\title{
Research Article Synchronization of Discrete-Time Stochastic Neural Networks with Random Delay
}

\author{
Haibo Bao and Jinde Cao \\ Department of Mathematics, Southeast University, Nanjing 210096, China \\ Correspondence should be addressed to Jinde Cao, jdcao@seu.edu.cn
}

Received 2 December 2010; Accepted 25 January 2011

Academic Editor: Recai Kilic

Copyright (C 2011 H. Bao and J. Cao. This is an open access article distributed under the Creative Commons Attribution License, which permits unrestricted use, distribution, and reproduction in any medium, provided the original work is properly cited.

By using a Lyapunov-Krasovskii functional method and the stochastic analysis technique, we investigate the problem of synchronization for discrete-time stochastic neural networks (DSNNs) with random delays. A control law is designed, and sufficient conditions are established that guarantee the synchronization of two identical DSNNs with random delays. Compared with the previous works, the time delay is assumed to be existent in a random fashion. The stochastic disturbances are described in terms of a Brownian motion and the time-varying delay is characterized by introducing a Bernoulli stochastic variable. Two examples are given to illustrate the effectiveness of the proposed results. The main contribution of this paper is that the obtained results are dependent on not only the bound but also the distribution probability of the time delay. Moreover, our results provide a larger allowance variation range of the delay, and are less conservative than the traditional delay-independent ones.

\section{Introduction}

Synchronization is one of the most important dynamic behavior of complex networks, which means if two or more systems have something in common, they will adjust each other to give rise to a common dynamical behavior. It has been found applications in many fields such as synchronous information exchange in the Internet WWW, crickets chirping in synchrony, rhythmic applause, and synchronous transfer of digital or analog signals in the communication networks.

Since the pioneering works of Pecora and Carroll [1, 2], the control and synchronization problems have become an active topic that attracts a lot of researchers' interest, including general complex dynamical networks [3-8], and the array of coupled neural networks with or without delays [6,9-11]. Several different approaches have been proposed for the synchronization of chaotic systems, such as linear and nonlinear feedback control [12, 13], adaptive control [14], impulsive control [15], and intermittent linear state feedback [16]. 
Itô-type stochastic systems are well known for their important impact on practical applications such as chemistry, biology, ecology, control, and information systems. In real complex networks, the signal transmission could be a noisy process brought by random fluctuations from the release of probabilistic causes such as neurotransmitters. Stochastic neural networks, as a special case of complex networks, have gained much more researchers' interests; see for example, $[4,5,13,15,17,18]$ and the references therein.

Discrete-time neural networks play a more and more important role in engineering application. As pointed out in [19-21], the discretization cannot preserve the dynamics of continuous-time counterpart even for a small sampling period. Recently, the synchronization problem for discrete-time networks has received more attention $[4,5,15,22]$.

Time delays occur frequently in practical situations, it can cause undesirable dynamic network behaviors such as oscillation and instability. Therefore, dynamical behavior [23, 24], especially synchronization problem for discrete-time neural networks with constant and time-varying delays has gained interesting research attention; see, for example, [4, 5, 15, 22]. It is worth mentioning that as a particular kind of time delays, random delays have also received much researchers' attention [25-31]. This is mainly because in many real systems, some values of the delay are very large, but the variation range of time delay taking such large values are very small. In this case, if only the variation range of time delay is employed to derive the criteria, the results may be somewhat more conservative.

Inspired by the above discussion, the aim of this paper is to study the synchronization problem for a class of DSNNs with random delay. The effect of both variation range and distribution probability of the time delay are taken into account in the proposed approach. The stochastic disturbances are described in terms of a Brownian motion, and the timevarying delay is characterized by introducing a Bernoulli stochastic variable. By employing a Lyapunov-Krasovskii functional, sufficient delay-distribution-dependent conditions are established in terms of linear matrix inequalities (LMIs) that guarantee the exponentially mean square synchronization of two identical DSNNs with random delays, which can be checked readily by Matlab toolbox.

This paper is organized as follows. In Section 2, the model formulation and some preliminaries are given. The main results are stated in Section 3. Two illustrative examples are given to demonstrate the effectiveness of the proposed results in Section 4. Finally, the conclusions are made in Section 5.

\subsection{Notation}

Throughout this paper, $\mathbb{R}^{n}$ and $\mathbb{R}^{n \times m}$, respectively, denote the $n$-dimensional Euclidean and the set of all $n \times m$ matrices. $I$ is the identity matrix of appropriate dimensions. The superscript " $T$ " denotes matrix transposition. The notation $X>0$ (resp., $X \geq 0$ ), where $X$ is a real symmetric matrix, means $X$ is positive definite (resp., positive semidefinite). $|\cdot|$ is the Euclidean norm in $\mathbb{R}^{n}$. If $A$ is a matrix, $\|A\|$ denotes its operator norm. That is, $\|A\|=\sup \{|A x|:|x|=1\}=\sqrt{\lambda_{\max }\left(A^{T} A\right)}$, where $\lambda_{\max }(A)$ (resp., $\lambda_{\min }(A)$ ) means the largest (resp., smallest) eigenvalue of $A$. $\mathbb{Z}_{\geq 0}$ denotes the set including zero and positive integers. The asterisk ${ }^{*}$ in a matrix is used to denote term that is induced by symmetry. $\mathbb{E}\{\cdot\}$ denotes the expectation. Moreover, let $\left(\Omega, \mathcal{F},\left\{\mathcal{F}_{t}\right\}_{t \geq 0}, D\right)$ be a complete probability space with a filtration $\left\{\mathcal{F}_{t}\right\}_{t \geq 0}$ satisfying the usual conditions. Denote by $\mathcal{L}_{\mathscr{F}_{0}}^{2}\left([-\tau, 0], \mathbb{R}^{n}\right)$ the family of all $\mathcal{F}_{0}$-measurable $C\left([-\tau, 0]: \mathbb{R}^{n}\right)$-valued random variables $\phi=\{\phi(s),-\tau \leq s \leq 0\}$ with the norm $\|\phi\|=\sup _{-\tau \leq s \leq 0}|\phi(s)|^{2}<\infty$. 


\section{Problem Formulation}

Consider the following $n$-neuron DSNNs with time delay

$$
x(k+1)=A x(k)+B \tilde{F}(x(k))+D \tilde{G}(x(k-\tau(k))),
$$

where $x(k)=\left(x_{1}(k), x_{2}(k), \ldots, x_{n}(k)\right)^{T} \in \mathbb{R}^{n}$ is the state vector associated with the $n$ neurons, $A=\operatorname{diag}\left(a_{1}, a_{2}, \ldots, a_{n}\right)$ with $\left|a_{i}\right|<1 ; B=\left(b_{i j}\right)_{n \times n}, D=\left(d_{i j}\right)_{n \times n}$ denote the connection weights matrix and the delayed connection weights matrix, respectively, $\widetilde{F}(x(k))=\left[\tilde{f}_{1}\left(x_{1}(k)\right)\right.$, $\left.\tilde{f}_{2}\left(x_{2}(k)\right), \ldots, \tilde{f}_{n}\left(x_{n}(k)\right)\right]^{T}$ and $\tilde{G}(x(k))=\left[\tilde{g}_{1}\left(x_{1}(k)\right), \widetilde{g}_{2}\left(x_{2}(k)\right), \ldots, \widetilde{g}_{n}\left(x_{n}(k)\right)\right]^{T}$ denote the neuron activation functions; $\tau(k)$ denotes the time-varying delay.

In this paper, we consider the model (2.1) as the drive system, and the noise-perturbed response system is given as

$$
\widehat{x}(k+1)=A \widehat{x}(k)+B \tilde{F}(\widehat{x}(k))+D \tilde{G}(\widehat{x}(k-\tau(k)))+u(k)+\sigma(k, \widehat{x}(k)-x(k)) \omega(k),
$$

where $u(k)=\left(u_{1}(k), u_{2}(k), \ldots, u_{n}(k)\right)^{T} \in \mathbb{R}^{n}$ is the state feedback controller given to achieve the global exponental synchronization between the drive-response system; $\sigma: \mathbb{R} \times \mathbb{R}^{n} \rightarrow \mathbb{R}^{n}$ is a continuous function, $\omega(k)$ is a scalar Wiener process on a probability space $(\Omega, \mathcal{F}, D)$ with

$$
\mathbb{E}\{\omega(k)\}=0, \quad \mathbb{E}\left\{\omega^{2}(k)\right\}=1, \quad \mathbb{E}\{\omega(i) \omega(j)\}=0 \quad(i \neq j) .
$$

Throughout this paper, the following assumptions are made.

Assumption 2.1. For $i \in\{1,2, \ldots, n\}$, the neuron activation functions $\tilde{f}_{i}(\cdot)$ and $\tilde{g}_{i}(\cdot)$ are continuous and bounded and satisfy the following conditions:

$$
\begin{gathered}
l_{i} \leq \frac{\tilde{f}_{i}\left(s_{1}\right)-\tilde{f}_{i}\left(s_{2}\right)}{s_{1}-s_{2}} \leq L_{i}, \\
\omega_{i} \leq \frac{\tilde{g}_{i}\left(s_{1}\right)-\tilde{g}_{i}\left(s_{2}\right)}{s_{1}-s_{2}} \leq W_{i}, \quad \forall s_{1}, s_{2} \in \mathbb{R}\left(s_{1} \neq s_{2}\right), i=1,2, \ldots, n .
\end{gathered}
$$

As first discussed in [32], for brevity of the following representation, we denote

$$
\begin{gathered}
\mathcal{C}_{1}=\operatorname{diag}\left\{l_{1} L_{1}, \ldots, l_{n} L_{n}\right\}, \quad \mathcal{C}_{2}=\operatorname{diag}\left\{-\frac{l_{1}+L_{1}}{2}, \ldots,-\frac{l_{n}+L_{n}}{2}\right\}, \\
\mathscr{L}_{1}=\operatorname{diag}\left\{\omega_{1} W_{1}, \ldots, \omega_{n} W_{n}\right\}, \quad \mathscr{L}_{2}=\operatorname{diag}\left\{-\frac{\omega_{1}+W_{1}}{2}, \ldots,-\frac{\omega_{n}+W_{n}}{2}\right\} .
\end{gathered}
$$

Assumption 2.2. $\sigma(k, x(k))$ is a continuous function satisfying $\sigma(0,0)=0$ and

$$
\sigma(k, x(k))^{T} \sigma(k, x(k)) \leq \rho x(k)^{T} x(k),
$$

where $\rho>0$ is a known constant scalar. 
Assumption 2.3. The time delay $\tau(k)$ is bounded, $0 \leq \tau_{m}<\tau(k) \leq \tau_{M}$, and its probability distribution can be observed; that is, suppose that $\tau(k)$ takes values in $\left[\tau_{m}: \tau_{0}\right]$ or $\left(\tau_{0}: \tau_{M}\right]$ and $\operatorname{Prob}\left\{\tau(k) \in\left[\tau_{m}: \tau_{0}\right]\right\}=\delta_{0}$, where $\tau_{m} \leq \tau_{0}<\tau_{M}$, and $0 \leq \delta_{0} \leq 1$.

Remark 2.4. It is noted that the introduction of binary stochastic variable was first introduced in [25] and then successfully used in [26-31]. Under the Assumption 2.3, we know that $\delta_{0}$ is dependent on the values of $\tau_{m}, \tau_{0}, \tau_{M}$. In addition, $\operatorname{Prob}\left\{\tau(k) \in\left(\tau_{0}: \tau_{M}\right]\right\}=1-\delta_{0} \triangleq \bar{\delta}_{0}$.

In order to describe the probability distribution of the time delay, we define two sets

$$
\boldsymbol{B}_{1}=\left\{k \mid \tau(k) \in\left[\tau_{m}, \tau_{0}\right]\right\}, \quad \boldsymbol{B}_{2}=\left\{k \mid \tau(k) \in\left(\tau_{0}, \tau_{M}\right]\right\},
$$

where $\tau_{0}$ is an integer satisfying $\tau_{m} \leq \tau_{0}<\tau_{M}$. Define two mapping functions

$$
\tau_{1}(k)=\left\{\begin{array}{ll}
\tau(k), & k \in \mathcal{B}_{1}, \\
\tau_{m}, & \text { else, }
\end{array} \quad \tau_{2}(k)= \begin{cases}\tau(k), & k \in \mathcal{B}_{2} \\
\tau_{0}, & \text { else. }\end{cases}\right.
$$

It follows from (2.7) that $\boldsymbol{B}_{1} \cup \boldsymbol{B}_{2}=\mathbb{Z}_{\geq 0}$ and $\boldsymbol{B}_{1} \cap \boldsymbol{B}_{2}=\emptyset$. From (2.8), it can be seen that $k \in \boldsymbol{B}_{1}$ implies the event $\tau(k) \in\left[\tau_{m}: \tau_{0}\right]$ occurs and $k \in B_{2}$ implies the event $\tau(k) \in\left(\tau_{0}: \tau_{M}\right]$ occurs. Defining a stochastic variable as

$$
\delta(k)= \begin{cases}1, & k \in B_{1}, \\ 0, & k \in B_{2},\end{cases}
$$

then system (2.1) and (2.2) can be equivalently rewritten as

$$
\begin{aligned}
x(k+1)= & A x(k)+B \tilde{F}(x(k))+\delta(k) D \tilde{G}\left(x\left(k-\tau_{1}(k)\right)\right)+(1-\delta(k)) D \tilde{G}\left(x\left(k-\tau_{2}(k)\right)\right) \\
\widehat{x}(k+1)= & A \widehat{x}(k)+B \widetilde{F}(\widehat{x}(k))+\delta(k) D \tilde{G}\left(\widehat{x}\left(k-\tau_{1}(k)\right)\right)+(1-\delta(k)) D \tilde{G}\left(\widehat{x}\left(k-\tau_{2}(k)\right)\right)+u(k) \\
& +\sigma(k, \widehat{x}(k)-x(k)) \omega(k) .
\end{aligned}
$$

It is further assumed that the variables $\delta(k)$ and $\omega(k)$ are mutually independent.

Remark 2.5. Under the Assumption 2.3 and the definition of $\delta(k)$, it can be seen that $\delta(k)$ is a Bernoulli distributed white sequence with $\operatorname{Prob}\{\delta(k)=1\}=\mathbb{E}\{\delta(k)\}=\delta_{0}$ and $\operatorname{Prob}\{\delta(k)=$ $0\}=\bar{\delta}_{0}$. Furthermore, we can show that $\mathbb{E}\left\{\delta(k)-\delta_{0}\right\}=0$ and $\mathbb{E}\left\{\left(\delta(k)-\delta_{0}\right)^{2}\right\}=\delta_{0} \bar{\delta}_{0}$. 

follows:

Letting $e(k)=\hat{x}(k)-x(k)$ be the synchronization error, we can derive error system as

$$
\begin{aligned}
e(k+1)= & A e(k)+B F(e(k))+\delta(k) D G\left(e\left(k-\tau_{1}(k)\right)\right)+(1-\delta(k)) D G\left(e\left(k-\tau_{2}(k)\right)\right) \\
& +\sigma(k, e(k)) \omega(k)+u(k),
\end{aligned}
$$

where

$$
\begin{aligned}
& F(e(k))=\left[f_{1}\left(e_{1}(k)\right), f_{2}\left(e_{2}(k)\right), \ldots, f_{n}\left(e_{n}(k)\right)\right]^{T}=\tilde{F}(\widehat{x}(k))-\tilde{F}(x(k)), \\
& G(e(k))=\left[g_{1}\left(e_{1}(k)\right), g_{2}\left(e_{2}(k)\right), \ldots, g_{n}\left(e_{n}(k)\right)\right]^{T}=\tilde{G}(\widehat{x}(k))-\tilde{G}(x(k)) .
\end{aligned}
$$

The initial condition associated with (2.12) is given as

$$
e(s)=\phi(s), \quad s \in \mathbb{N}\left[-\tau_{M}, 0\right]
$$

where $\phi(s) \in \mathcal{L}_{\bar{f}_{0}}^{2}\left(\left[-\tau_{M}, 0\right], \mathbb{R}^{n}\right)$, and $\mathbb{N}\left[-\tau_{M}, 0\right]=\left\{-\tau_{M},-\tau_{M}+1, \ldots, 0\right\}$.

Let $e(k, \phi)$ be the state trajectory of system (2.12) under the initial condition. It is obvious that $e(k, 0)=0$ is a trivial solution of DSNNs (2.12).

Definition 2.6. The drive system (2.10) and the response system (2.11) are said to be exponentially synchronized if, for a suitably designed feedback controller, the trivial solution of the error system (2.12) is globally exponentially stable in the mean square. That is, there exist constants $\vartheta>0$, and $v \in(0,1)$ such that for sufficiently integer $T>0$, the inequality

$$
\mathbb{E}|e(k)|^{2} \leq \vartheta v^{k} \sup _{s \in \mathbb{N}\left[-\tau_{M}, 0\right]} \mathbb{E}|e(s)|^{2}
$$

holds for all $k>T$.

For brevity of the following analysis, we denote $e(k), e\left(k-\tau_{1}(k)\right), e\left(k-\tau_{2}(k)\right), \delta(k)$, $1-\delta(k), \omega(k)$ by $e_{k}, e_{\tau, 1}, e_{\tau, 2}, \delta_{k}, \bar{\delta}_{k}, \omega_{k}$, respectively. Then, (2.12) can be rearranged as

$$
e_{k+1}=A e_{k}+B F\left(e_{k}\right)+\delta_{k} D G\left(e_{\tau, 1}\right)+\bar{\delta}_{k} D G\left(e_{\tau, 2}\right)+\sigma\left(k, e_{k}\right) \omega_{k}+u(k) .
$$

\section{Main Results}

In order to realize the synchronization between the drive system (2.10) and the noiseperturbed response system (2.11) with different initial conditions, we will design a suitable feedback controller and develop theoretical results of the synchronization scheme. In fact, if the trivial solution of the controlled error system (2.12) is exponentially stable in the mean square, (2.10) and (2.11) can achieve globally synchronization.

For the noised-perturbed response system (2.11), the feedback controller $u(k)$ is designed as

$$
u(k)=K e(k)+\delta_{0} K_{1} e\left(k-\tau_{1}(k)\right)+\left(1-\delta_{0}\right) K_{1} e\left(k-\tau_{2}(k)\right),
$$

where $K$ and $K_{1}$ are the feedback gains to be determined. 
Theorem 3.1. Suppose that Assumptions 2.1-2.3 hold, and let the state-feedback controller (3.1) be given. The noised-perturbed response system (2.11) is globally exponentially synchronized with the drive model (2.10) if there exist three positive definite matrices $P, Q_{1}, Q_{2}$, three diagonal matrices $\Lambda_{i}=\operatorname{diag}\left(\lambda_{i 1}, \lambda_{i 2}, \ldots, \lambda_{i n}\right)>0(i=1,2,3)$, two real matrices $R, R_{1}$ and a scalar $\lambda^{*}>0$ such that the following two LMIs hold:

$$
\Omega=\left[\begin{array}{ccccccc}
\Xi_{1} & 0 & 0 & -\Lambda_{1} \mathcal{C}_{2} & 0 & 0 & A^{T} P+R^{T} \\
* & \Xi_{2} & 0 & 0 & -\Lambda_{2} \mathscr{\ell}_{2} & 0 & \sqrt{\delta_{0}} R_{1}^{T} \\
* & * & \Xi_{3} & 0 & 0 & -\Lambda_{3} \mathcal{L}_{2} & \sqrt{\bar{\delta}_{0}} R_{1}^{T} \\
* & * & * & -\Lambda_{1} & 0 & 0 & B^{T} P \\
* & * & * & * & -\Lambda_{2} & 0 & \sqrt{\delta_{0}} D^{T} P \\
* & * & * & * & * & -\Lambda_{3} & \sqrt{\bar{\delta}_{0}} D^{T} P \\
* & * & * & * & * & * & -P
\end{array}\right]<0,
$$

where $\Xi_{1}=-P+\lambda^{*} \rho I+\left(\tau_{0}-\tau_{m}+1\right) Q_{1}+\left(\tau_{M}-\tau_{0}+2\right) Q_{2}-\Lambda_{1} \mathcal{C}_{1}, \Xi_{2}=-Q_{1}-\Lambda_{2} \mathscr{\ell}_{1}, \Xi_{3}=-Q_{2}-\Lambda_{3} \mathscr{\ell}_{1}$.

And then the feedback gains can be designed as

$$
K=P^{-1} R, \quad K_{1}=P^{-1} R_{1} .
$$

Proof. See the Appendix B.

Remark 3.2. We would like to point out that there is still enough room to improve the result. Because of (1) in real-time systems, time delays always exist in a stochastic fashion, so we also can consider the time delays satisfy other distributions. (2) And we can also extend this method to the dynamics of discrete-time stochastic complex networks. (3) The results can be improved by combining with delay-fractioning method to reduce conservatism.

Remark 3.3. Our results are less conservative than some other existed results because they are dependent on not only the bound but also the distribution probability of the time delays, and we obtain a larger allowance variation range of the delay, while the delay-fractioning or delay-partitioning approach [22] can reduce conservatism lie in the methods increase the number of fraction of the time delay. The delay-fractioning approach is a very effective approach to reduce conservatism. And we will combine with the free-weighting methods or delay partitioning approaches to reduce the conservativeness of the results in the future.

Remark 3.4. When $\delta(k) \equiv 1\left(\forall k \in \mathbb{Z}_{\geq 0}\right)$, which means $\tau_{1}(k) \equiv \tau(k),(2.10)$ reduces to (2.1). By setting $\delta_{0}=1, Q_{2}=0$ in Theorem 3.1 and deleting the third and sixth rows and the corresponding third and sixth columns of (3.2), we can obtain the following results. 
Now, (2.11) reduces to

$$
\begin{aligned}
\widehat{x}(k+1)= & A \widehat{x}(k)+B \tilde{F}(\widehat{x}(k))+D \tilde{G}(\widehat{x}(k-\tau(k)))+u(k) \\
& +\sigma(k, \widehat{x}(k)-x(k)) \omega(k) .
\end{aligned}
$$

Equation (2.16) reduces to

$$
e_{k+1}=A e_{k}+B F\left(e_{k}\right)+D G\left(e_{\tau}\right)+\sigma\left(k, e_{k}\right) \omega_{k}+u(k) .
$$

Corollary 3.5. Suppose that Assumptions 2.1-2.2 hold, and let the state-feedback controller (3.1) be given. The noised-perturbed response system (3.5) is globally exponentially synchronized with the drive model (2.1) if there exist two positive definite matrices $P, Q_{1}$, two diagonal matrices $\Lambda_{i}=$ $\operatorname{diag}\left(\lambda_{i 1}, \lambda_{i 2}, \ldots, \lambda_{i n}\right)>0(i=1,2)$, a real matrix $R$, and a scalar $\lambda^{*}>0$, such that the following two LMIs hold:

$$
\Omega_{2}=\left[\begin{array}{ccccc}
\Gamma_{1} & 0 & -\Lambda C_{2} & 0 & A^{T} P+R^{T} \\
* & \Gamma_{2} & 0 & -\Lambda_{2} \ell_{2} & R_{1}^{T} \\
* & * & -\Lambda_{1} & 0 & B^{T} P \\
* & * & * & -\Lambda_{2} & D^{T} P \\
* & * & * & * & -P
\end{array}\right]<0,
$$

where $\Gamma_{1}=-P+\lambda^{*} \rho I+\left(\tau_{0}-\tau_{m}+1\right) Q_{1}-\Lambda_{1} \mathcal{C}_{1}, \Gamma_{2}=-Q_{1}-\Lambda_{2} \mathscr{\ell}_{1}$.

And then the feedback gains can be designed as

$$
K=P^{-1} R \text {. }
$$

Remark 3.6. The model proposed in this paper takes some well-studied models as special cases such as the model given in [4].

If we neglect the effect of the stochastic term $\omega_{k}$ in (2.11), then (2.11) reduces to

$$
\begin{aligned}
\widehat{x}(k+1)= & A \widehat{x}(k)+B \widetilde{F}(\widehat{x}(k))+\delta(k) D \tilde{G}\left(\widehat{x}\left(k-\tau_{1}(k)\right)\right) \\
& +(1-\delta(k)) D \tilde{G}\left(\widehat{x}\left(k-\tau_{2}(k)\right)\right)+u(k) .
\end{aligned}
$$

Equation (2.16) reduces to

$$
e_{k+1}=A e_{k}+B F\left(e_{k}\right)+\delta_{k} D G\left(e_{\tau, 1}\right)+\bar{\delta}_{k} D G\left(e_{\tau, 2}\right)+u(k)
$$

In this case, we have the following results. 
Corollary 3.7. Suppose that Assumptions 2.1 and 2.3 hold, and let the state-feedback controller (3.1) be given. The response system (3.10) is globally exponentially synchronized with the drive model (2.10) if there exist three positive definite matrices $P, Q_{1}, Q_{2}$, three diagonal matrices $\Lambda_{i}=$ $\operatorname{diag}\left(\lambda_{i 1}, \lambda_{i 2}, \ldots, \lambda_{i n}\right)>0(i=1,2,3)$, two real matrices $R, R_{1}$, such that the following LMI holds:

$$
\Omega_{3}=\left[\begin{array}{ccccccc}
\Pi_{1} & 0 & 0 & -\Lambda_{1} \mathcal{C}_{2} & 0 & 0 & A^{T} P+R^{T} \\
* & \Pi_{2} & 0 & 0 & -\Lambda_{2} \mathscr{\ell}_{2} & 0 & \sqrt{\delta_{0}} R_{1}^{T} \\
* & * & \Pi_{3} & 0 & 0 & -\Lambda_{3} \mathcal{L}_{2} & \sqrt{\bar{\delta}_{0}} R_{1}^{T} \\
* & * & * & -\Lambda_{1} & 0 & 0 & B^{T} P \\
* & * & * & * & -\Lambda_{2} & 0 & \sqrt{\delta_{0}} D^{T} P \\
* & * & * & * & * & -\Lambda_{3} & \sqrt{\bar{\delta}_{0}} D^{T} P \\
* & * & * & * & * & * & -P
\end{array}\right]<0,
$$

where $\Pi_{1}=-P+\left(\tau_{0}-\tau_{m}+1\right) Q_{1}+\left(\tau_{M}-\tau_{0}+2\right) Q_{2}-\Lambda_{1} \mathcal{C}_{1}, \Pi_{2}=-Q_{1}-\Lambda_{2} \mathfrak{\ell}_{1}, \Pi_{3}=-Q_{2}-\Lambda_{3} \mathfrak{l}_{1}$. And then the feedback gains can be designed as

$$
K=P^{-1} R, \quad K_{1}=P^{-1} R_{1} .
$$

\section{Two Numerical Examples}

Two numerical examples are presented to demonstrate the effectiveness of our results.

Example 4.1. Consider the DSNNs (2.10) with the following parameters:

$$
\begin{gathered}
A=\left[\begin{array}{ccc}
0.4 & 0 & 0 \\
0 & 0.5 & 0 \\
0 & 0 & 0.4
\end{array}\right], \quad B=\left[\begin{array}{ccc}
0.3 & -0.1 & 0.2 \\
0 & -0.3 & 0.2 \\
-0.1 & -0.1 & -0.2
\end{array}\right], \quad D=\left[\begin{array}{ccc}
0.2 & 0.1 & 0.1 \\
-0.2 & 0.3 & 0.1 \\
0.1 & -0.2 & 0.3
\end{array}\right], \\
f_{1}(s)=\tanh (0.6 s)-0.2 \sin (s), \quad f_{2}(s)=\tanh (-0.4 s), \quad f_{3}(s)=\tanh (-0.2 s), \\
g_{1}(s)=\tanh (-0.4 s)+0.2 \sin (s), \quad g_{2}(s)=\tanh (0.2 s), \quad g_{3}(s)=\tanh (0.4 s),
\end{gathered}
$$

It is easy to verify that

$$
\begin{gathered}
\mathcal{C}_{1}=\operatorname{diag}(-0.08,0,0), \quad \mathcal{C}_{2}=\operatorname{diag}(0.1,0.2,0.1), \\
\mathscr{L}_{1}=\operatorname{diag}(-0.06,0,0), \quad \mathscr{L}_{2}=\operatorname{diag}(0.06,-0.1,-0.2) .
\end{gathered}
$$


Setting $\tau_{m}=1, \tau_{0}=2, \tau_{M}=5$, and $\delta_{0}=0.89, \rho=0.1$ in Theorem 3.1, we can verify by Matlab toolbox that LMIs (3.2) and (3.3) are solved and the solutions are given as follows:

$$
\begin{aligned}
& P=\left[\begin{array}{ccc}
15.7114 & -0.8610 & 0.1575 \\
-0.8610 & 11.9585 & 1.0510 \\
0.1575 & 1.0510 & 14.8306
\end{array}\right], \quad Q_{1}=\left[\begin{array}{ccc}
1.9720 & -0.1597 & 0.0202 \\
-0.1597 & 2.0645 & 0.2055 \\
0.0202 & 0.2055 & 2.2099
\end{array}\right], \\
& Q_{2}=\left[\begin{array}{ccc}
0.6299 & -0.0191 & 0.0012 \\
-0.0191 & 0.3460 & 0.0251 \\
0.0012 & 0.0251 & 0.5228
\end{array}\right], \quad \Lambda_{1}=\left[\begin{array}{ccc}
369.9501 & 0 & 0 \\
0 & 369.9501 & 0 \\
0 & 0 & 369.9501
\end{array}\right] \text {, } \\
& \Lambda_{2}=\left[\begin{array}{ccc}
369.9501 & 0 & 0 \\
0 & 369.9501 & 0 \\
0 & 0 & 369.9501
\end{array}\right], \quad \Lambda_{3}=\left[\begin{array}{ccc}
369.9501 & 0 & 0 \\
0 & 369.9501 & 0 \\
0 & 0 & 369.9501
\end{array}\right] \text {, } \\
& R=\left[\begin{array}{ccc}
-5.7416 & 0.3131 & 0.0554 \\
-0.0464 & -6.5348 & -0.3567 \\
0.0554 & -0.3567 & -6.4303
\end{array}\right], \quad R_{1}=\left[\begin{array}{ccc}
0.1675 & -0.2223 & -0.1017 \\
-0.2223 & -0.4297 & -0.2061 \\
-0.1017 & -0.2061 & -0.7492
\end{array}\right], \quad \lambda^{*}=16.0757 \\
& K=P^{-1} R=\left[\begin{array}{ccc}
-0.3656 & -0.0103 & 0.0084 \\
-0.0008 & -0.5485 & 0.0089 \\
0.0077 & 0.0419 & -0.4343
\end{array}\right], \quad K_{1}=P^{-1} R_{1}=\left[\begin{array}{ccc}
0.0098 & -0.0160 & -0.0067 \\
-0.0174 & -0.0361 & -0.0134 \\
-0.0057 & -0.0112 & -0.495
\end{array}\right]
\end{aligned}
$$

Therefore, it can be seen from Theorem 3.1, the response system (2.11) is globally exponentially synchronized with the drive system (2.10). The result is further verified by the simulation results given by Figures 1 and 2. Figures 1(a), 1(b), and 1(c) represent the trajectories of $x_{1}(k), \widehat{x}_{1}(k), x_{2}(k), \hat{x}_{2}(k)$, and $x_{3}(k), \widehat{x}_{3}(k)$, respectively, and the red line represents the drive system state, the blue line stands for the response system state, and the initial conditions are taken as $x(k)=[1.0,2.5,0.5]^{T}, \widehat{x}(k)=[-0.5,-0.5,-0.5]^{T}$. Figure 2 shows that the error state goes to zero after a short period time. Furthermore, if we increase $\delta_{0}$, the maximum allowance value of $\tau_{M}$ will increase subsequently. Specially, if $\delta_{0}=0.99$, we get the maximum allowance value of $\tau_{M}=13$.

Example 4.2. Consider the DSNNs (2.10) with the following parameters:

$$
\begin{gathered}
A=\left[\begin{array}{cc}
-0.1 & 0 \\
0 & 0.4
\end{array}\right], \quad B=\left[\begin{array}{rr}
0.1 & -0.2 \\
0 & -0.1
\end{array}\right], \quad D=\left[\begin{array}{cc}
0 & 0.2 \\
0.2 & -0.1
\end{array}\right], \quad \rho=0.02, \\
f_{1}(s)=\sin (0.2 s)-0.6 \cos (s), \quad f_{2}(s)=\tanh (-0.4 s), \\
g_{1}(s)=\tanh (0.83 s)+0.6 \cos (s), \quad g_{2}(s)=\tanh (0.2 s) .
\end{gathered}
$$




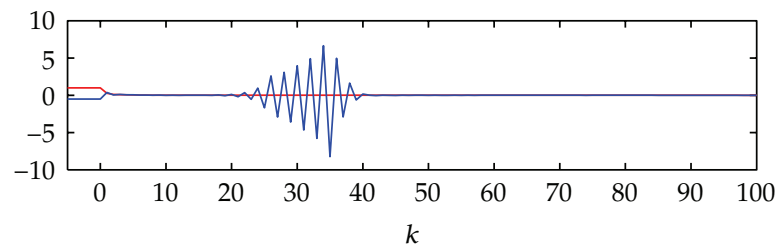

(a)

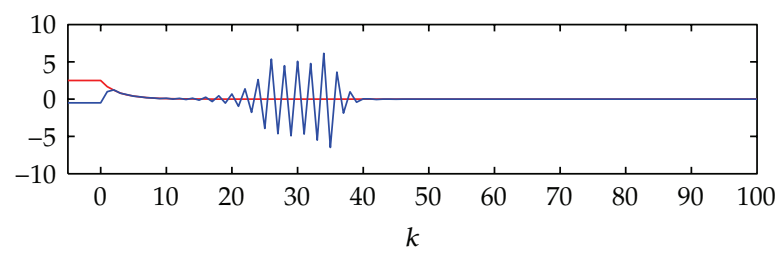

(b)

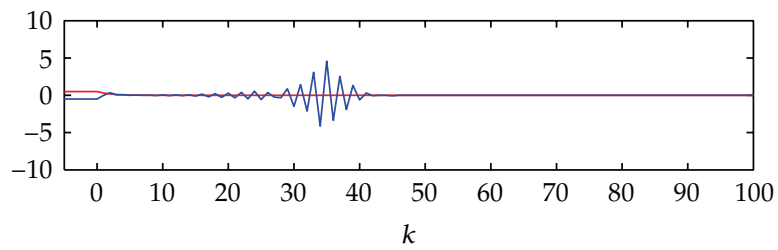

(c)

Figure 1: State trajectories of $x(k), \widehat{x}(k)$.

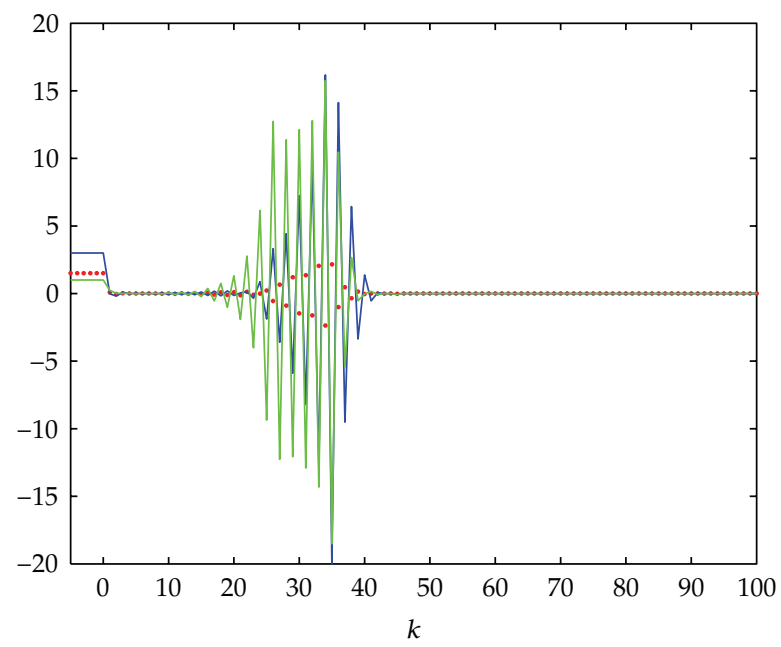

$$
\begin{array}{r}
e_{1} \\
-e_{2} \\
-e_{3}
\end{array}
$$

Figure 2: Error state trajectories of $e(k)$.

It is easy to verify that

$$
\mathcal{C}_{1}=\operatorname{diag}(-0.64,0), \quad \mathcal{C}_{2}=\operatorname{diag}(0,0.2), \quad \mathscr{H}_{1}=\operatorname{diag}(-0.6,0), \quad \mathscr{\ell}_{2}=\operatorname{diag}(-0.2,-0.1) \text {. }
$$




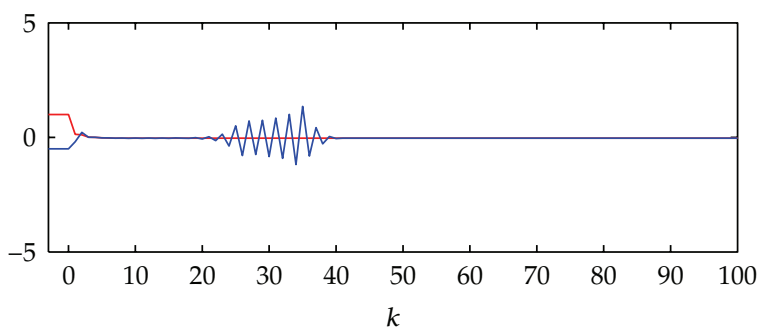

(a)

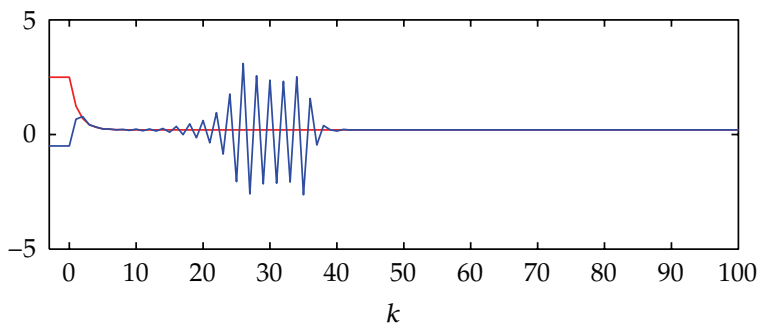

(b)

Figure 3: State trajectories of $x(k), \widehat{x}(k)$.

Setting $\tau_{m}=1, \tau_{0}=2, \tau_{M}=3$, and $\delta_{0}=0.85$ in Theorem 3.1, we can verify by Matlab toolbox that LMIs (3.2) and (3.3) are solved and the solutions are given below:

$$
\begin{gathered}
P=\left[\begin{array}{cc}
48.7972 & -2.3255 \\
-2.3255 & 42.7033
\end{array}\right], \quad Q_{1}=\left[\begin{array}{cc}
4.2255 & -0.4620 \\
-0.4620 & 8.3487
\end{array}\right], \quad Q_{2}=\left[\begin{array}{cc}
4.0513 & -0.1026 \\
-0.1026 & 1.8888
\end{array}\right], \\
\Lambda_{1}=\left[\begin{array}{cc}
880.7735 & 0 \\
0 & 880.7735
\end{array}\right], \quad \Lambda_{2}=\left[\begin{array}{cc}
880.7735 & 0 \\
0 & 880.7735
\end{array}\right], \quad \Lambda_{3}=\left[\begin{array}{cc}
880.7735 & 0 \\
0 & 880.7735
\end{array}\right], \\
R=\left[\begin{array}{cc}
4.8936 & -0.7368 \\
-0.7368 & -17.8357
\end{array}\right], \quad R_{1}=\left[\begin{array}{cc}
0.0860 & -1.2452 \\
-1.2452 & 0.3222
\end{array}\right], \quad \lambda^{*}=49.8783, \\
K=P^{-1} R=\left[\begin{array}{cc}
0.0997 & -0.0351 \\
-0.0118 & -0.4196
\end{array}\right], \quad K_{1}=P^{-1} R_{1}=\left[\begin{array}{cc}
0.0004 & -0.0252 \\
-0.0291 & 0.0062
\end{array}\right] .
\end{gathered}
$$

Therefore, it can be seen from Theorem 3.1, the response system (2.11) is globally exponentially synchronized with the drive system (2.10). The result is further verified by the simulation results given by Figures 3 and 4. Figures 3(a) and 3(b) represent the trajectories of $x_{1}(k), \hat{x}_{1}(k)$ and $x_{2}(k), \hat{x}_{2}(k)$, respectively, and the red line represents the drive system state, the blue line stands for the response system state, the initial conditions are taken as $x(k)=[1.0,2.5]^{T}, \widehat{x}(k)=[-0.5,-0.5]^{T}$. Figure 4 shows that the error state goes to zero after a short period time. 


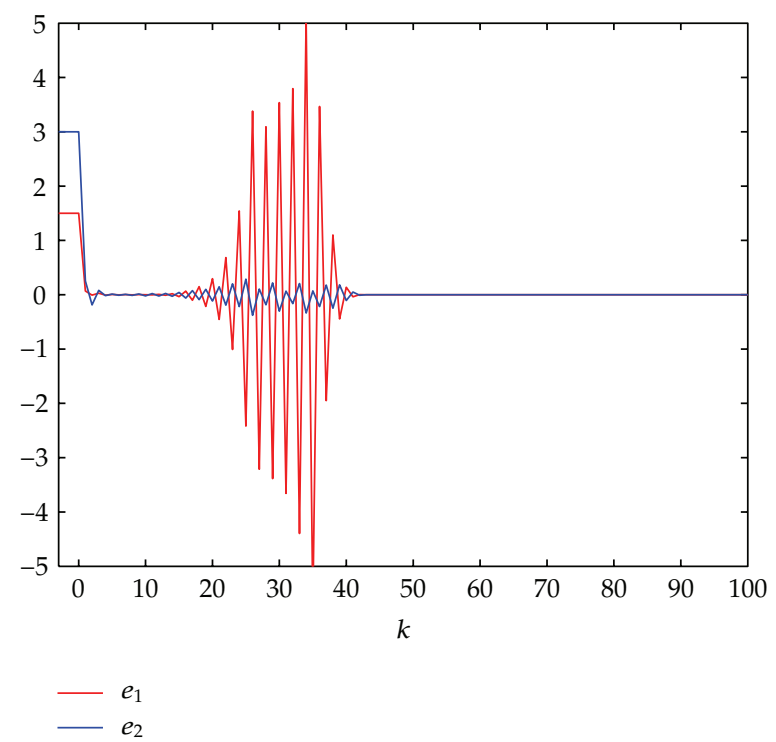

Figure 4: Error state trajectories of $e(k)$.

\section{Conclusions}

In recent years, synchronization in networks has become a hot research subject. However, the corresponding results are none for DSNNs with random delay. This paper has addressed the problem of exponential synchronization for the drive-response systems, where the drive system describes a class of discrete-time stochastic neural networks (DSNNs) with random delays and the response system is disturbed by some stochastic motions. Sufficient conditions have been established in terms of LMIs, which can be checked readily by Matlab toolbox. Two examples have been given to illustrate the effectiveness of the proposed results.

It is worth noting that the following two interesting and important issues should be addressed in our future work. Firstly, within the same LMI framework, it is not difficult to extend our main results to the synchronization problem for DSNNs with randomly mixed time-varying delays. Another future work may be how to extend the obtained results to DSNNs with Markovian jump and so on.

\section{Appendices}

\section{A. Preliminary Lemmas}

To obtain our main results, we need the following lemmas.

Lemma A.1 (see [33]). Let $X \in \mathbb{R}^{n}, Y \in \mathbb{R}^{n}, \epsilon>0$, then we have

$$
X^{T} Y+Y^{T} X \leq \epsilon X^{T} X+\epsilon^{-1} Y^{T} Y
$$

Lemma A.2 (Schur Complement [34]). For a given matrix,

$$
S=\left[\begin{array}{ll}
S_{11} & S_{12} \\
S_{12}^{T} & S_{22}
\end{array}\right]<0
$$


is equivalent to any one of the following conditions:

(i) $S_{22}<0, S_{11}-S_{12} S_{22}^{-1} S_{12}^{T}<0$,
(ii) $S_{11}<0, S_{22}-S_{12}^{T} S_{11}^{-1} S_{12}<0$.

\section{B. Proof of Theorem 3.1}

For notation convenience, in the sequel, we denote

$$
\begin{gathered}
\mathcal{E}_{k}=\left[e_{k}^{T}, e_{k-1}^{T}, \ldots, e_{k-\tau(k)}^{T}\right], \\
\xi_{k}=\left[e_{k}^{T}, e_{\tau, 1}^{T}, e_{\tau, 2}^{T}, F^{T}\left(e_{k}\right), G^{T}\left(e_{\tau, 1}\right), G^{T}\left(e_{\tau, 2}\right)\right]^{T}, \\
\Psi=\left[(A+K), \sqrt{\delta_{0}} K_{1}, \sqrt{\bar{\delta}_{0}} K_{1}, B, \sqrt{\delta_{0}} D, \sqrt{\bar{\delta}_{0}} D\right] .
\end{gathered}
$$

Now, in order to ensure that (2.11) is globally exponentially synchronized with (2.10), we just need to show that the error system (2.12) or (2.16) is globally exponentially stable in the mean square. To this end, we construct the following Lyapunov-Krasovskii functional $V_{k}$ by

$$
V_{k}=V_{k, 1}+V_{k, 2}+V_{k, 3}
$$

where

$$
\begin{gathered}
V_{k, 1}=e_{k}^{T} P e_{k}, \\
V_{k, 2}=\sum_{i=k-\tau_{1}^{k}}^{k-1} e_{i}^{T} Q_{1} e_{i}+\sum_{i=\tau_{m}}^{\tau_{0}-1} \sum_{j=k-i}^{k-1} e_{j}^{T} Q_{1} e_{j}, \\
V_{k, 3}=\sum_{i=k-\tau_{2}^{k}}^{k-1} e_{i}^{T} Q_{2} e_{i}+\sum_{i=\tau_{0}+1}^{\tau_{M}-1} \sum_{j=k-i}^{k-1} e_{j}^{T} Q_{2} e_{j},
\end{gathered}
$$

where $P>0, Q_{1}>0, Q_{2}>0$.

Calculating the difference of $V_{k}$ along the solution of DSNN (2.16) and taking the mathematical expectation, we have

$$
\mathbb{E}\left\{\Delta V_{k}\right\}=\mathbb{E}\left\{\Delta V_{k, 1}\right\}+\mathbb{E}\left\{\Delta V_{k, 2}\right\}+\mathbb{E}\left\{\Delta V_{k, 3}\right\},
$$


where

$$
\begin{aligned}
& \mathbb{E}\left\{\Delta V_{k, 1}\right\}=\mathbb{E}\left\{\mathbb{E}\left\{V_{k+1,1}\right\} \mid \varepsilon_{k}-V_{k, 1}\right\} \\
&=\mathbb{E}\left\{e_{k}^{T}\left((A+K)^{T} P(A+K)-P\right) e_{k}+2 e_{k}^{T}(A+K)^{T} P B F\left(e_{k}\right)\right. \\
&+ 2 \delta_{0} e_{k}^{T}(A+K)^{T} P D G\left(e_{\tau, 1}\right)+2 \bar{\delta}_{0} e_{k}^{T}(A+K)^{T} P D G\left(e_{\tau, 2}\right) \\
&+2 \delta_{0} e_{k}^{T}(A+K)^{T} P K_{1} e_{\tau, 1}+2 \bar{\delta}_{0} e_{k}^{T}(A+K)^{T} P K_{1} e_{\tau, 2}+F^{T}\left(e_{k}\right) B^{T} P B F\left(e_{k}\right) \\
&+2 \delta_{0} F^{T}\left(e_{k}\right) B^{T} P D G\left(e_{\tau, 1}\right)+2 \bar{\delta}_{0} F^{T}\left(e_{k}\right) B^{T} P D G\left(e_{\tau, 2}\right)+\delta_{0} G^{T}\left(e_{\tau, 1}\right) D^{T} P D G\left(e_{\tau, 1}\right) \\
&+\bar{\delta}_{0} G^{T}\left(e_{\tau, 2}\right) D^{T} P D G\left(e_{\tau, 2}\right)+\sigma^{T}\left(k, e_{k}\right) P \sigma\left(k, e_{k}\right) \\
&+2 \delta_{0} F^{T}\left(e_{k}\right) B^{T} P K_{1} e_{\tau, 1}+2 \bar{\delta}_{0} F^{T}\left(e_{k}\right) B^{T} P K_{1} e_{\tau, 2}+2 \delta_{0}^{2} G^{T}\left(e_{\tau, 1}\right) D^{T} P K_{1} e_{\tau, 1} \\
&+\delta_{0} \bar{\delta}_{0} G^{T}\left(e_{\tau, 1}\right) D^{T} P K_{1} e_{\tau, 2}+\delta_{0} \bar{\delta}_{0} G^{T}\left(e_{\tau, 2}\right) D^{T} P K_{1} e_{\tau, 1} \\
&\left.+2 \bar{\delta}_{0}^{2} G^{T}\left(e_{\tau, 2}\right) D^{T} P K_{1} e_{\tau, 2}+\delta_{0}^{2} e_{\tau, 1}^{T} K_{1}^{T} P K_{1} e_{\tau, 1}+\bar{\delta}_{0}^{2} e_{\tau, 2}^{T} K_{1}^{T} P K_{1} e_{\tau, 2}\right\}
\end{aligned}
$$

$$
\mathbb{E}\left\{\Delta V_{k, 2}\right\}=\mathbb{E}\left\{\mathbb{E}\left\{V_{k+1,2}\right\} \mid \varepsilon_{k}-V_{k, 2}\right\}
$$

$$
\begin{aligned}
&= \mathbb{E}\left\{\left(\tau_{0}-\tau_{m}+1\right) e_{k}^{T} Q_{1} e_{k}-e_{\tau, 1}^{T} Q_{1} e_{\tau, 1}\right. \\
&\left.+\left(\sum_{i=k-\tau_{1}^{k+1}+1}^{k-\tau_{m}}+\sum_{i=k-\tau_{m}+1}^{k-1}-\sum_{i=k-\tau_{1}^{k}+1}^{k-1}-\sum_{i=k-\tau_{0}+1}^{k-\tau_{m}}\right) e_{i}^{T} Q_{1} e_{i}\right\} \\
& \leq \mathbb{E}\left\{\left(\tau_{0}-\tau_{m}+1\right) e_{k}^{T} Q_{1} e_{k}-e_{\tau, 1}^{T} Q_{1} e_{\tau, 1}\right\},
\end{aligned}
$$

$$
\begin{aligned}
\mathbb{E}\left\{\Delta V_{k, 3}\right\} & =\mathbb{E}\left\{\mathbb{E}\left\{V_{k+1,3}\right\} \mid \mathcal{\varepsilon}_{k}-V_{k, 3}\right\} \\
& \leq \mathbb{E}\left\{\left(\tau_{M}-\tau_{0}+2\right) e_{k}^{T} Q_{2} e_{k}-e_{\tau, 2}^{T} Q_{2} e_{\tau, 2}\right\} .
\end{aligned}
$$

It can be easily seen from Assumption 2.2 and (3.3) that

$$
\begin{aligned}
\mathbb{E}\left\{\sigma^{T}\left(k, e_{k}\right) P \sigma\left(k, e_{k}\right)\right\} & \leq \mathbb{E}\left\{\lambda_{\max }(P) \sigma^{T}\left(k, e_{k}\right) \sigma\left(k, e_{k}\right)\right\} \\
& \leq \mathbb{E}\left\{\lambda^{*} \rho e_{k}^{T} e_{k}\right\} .
\end{aligned}
$$


Combining (B.4)-(B.8), we obtain

$$
\begin{aligned}
\mathbb{E}\left\{\Delta V_{k}\right\} \leq \mathbb{E}\{ & e_{K}^{T}\left((A+K)^{T} P(A+K)-P+\left(\tau_{0}-\tau_{m}+1\right)+\left(\tau_{M}-\tau_{0}+2\right)+\lambda^{*} \rho I\right) e_{k} \\
& -e_{\tau, 1}^{T} Q_{1} e_{\tau, 1}-e_{\tau, 2}^{T} Q_{2} e_{\tau, 2}+2 e_{k}^{T}(A+K)^{T} P B F\left(e_{k}\right) \\
& +2 \delta_{0} e_{k}^{T}(A+K)^{T} P D G\left(e_{\tau, 1}\right)+2 \bar{\delta}_{0} e_{k}^{T}(A+K)^{T} P D G\left(e_{\tau, 2}\right)+2 \delta_{0} e_{k}^{T}(A+K)^{T} P K_{1} e_{\tau, 1} \\
& +2 \bar{\delta}_{0} e_{k}^{T}(A+K)^{T} P K_{1} e_{\tau, 2}+F^{T}\left(e_{k}\right) B^{T} P B F\left(e_{k}\right)+2 \delta_{0} F^{T}\left(e_{k}\right) B^{T} P D G\left(e_{\tau, 1}\right) \\
& +2 \bar{\delta}_{0} F^{T}\left(e_{k}\right) B^{T} P D G\left(e_{\tau, 2}\right)+2 \delta_{0} F^{T}\left(e_{k}\right) B^{T} P K_{1} e_{\tau, 1}+2 \bar{\delta}_{0} F^{T}\left(e_{k}\right) B^{T} P K_{1} e_{\tau, 2} \\
& +\delta_{0} G^{T}\left(e_{\tau, 1}\right) D^{T} P D G\left(e_{\tau, 1}\right)+\bar{\delta}_{0} G^{T}\left(e_{\tau, 2}\right) D^{T} P D G\left(e_{\tau, 2}\right)+2 \delta_{0}^{2} G^{T}\left(e_{\tau, 1}\right) D^{T} P K_{1} e_{\tau, 1} \\
& +\delta_{0} \bar{\delta}_{0} G^{T}\left(e_{\tau, 1}\right) D^{T} P K_{1} e_{\tau, 2}+\delta_{0} \bar{\delta}_{0} G^{T}\left(e_{\tau, 2}\right) D^{T} P K_{1} e_{\tau, 1} \\
& \left.+2 \bar{\delta}_{0}^{2} G^{T}\left(e_{\tau, 2}\right) D^{T} P K_{1} e_{\tau, 2}+\delta_{0}^{2} e_{\tau, 1}^{T} K_{1}^{T} P K_{1} e_{\tau, 1}+\bar{\delta}_{0}^{2} e_{\tau, 2}^{T} K_{1}^{T} P K_{1} e_{\tau, 2}\right\} \\
=\mathbb{E}\{ & \left.\xi_{k} \Omega_{0} \xi_{k}\right\}
\end{aligned}
$$

where

$$
\Omega_{0}=\left[\begin{array}{cccccc}
\Theta_{1} & \delta_{0}(A+K)^{T} P K_{1} & \bar{\delta}_{0}(A+K)^{T} P K_{1} & (A+K)^{T} P B & \delta_{0}(A+K)^{T} P D & \bar{\delta}_{0}(A+K)^{T} P D \\
* & \Theta_{2} & \delta_{0} \bar{\delta}_{0} K_{1}^{T} P K_{1} & \delta_{0} K_{1}^{T} P B & \delta_{0}^{2} K_{1}^{T} P D & \delta_{0} \bar{\delta}_{0} K_{1}^{T} P D \\
* & * & \Theta_{3} & \bar{\delta}_{0} K_{1}^{T} P B & \delta_{0} \bar{\delta}_{0} K_{1}^{T} P D & \bar{\delta}_{0}^{2} K_{1}^{T} P D \\
* & * & * & B^{T} P B & \delta_{0} B^{T} P D & \bar{\delta}_{0} B^{T} P D \\
* & * & * & * & \delta_{0} D^{T} P D & 0 \\
* & * & * & * & * & \bar{\delta}_{0} D^{T} P D
\end{array}\right]<0,
$$

where $\Theta_{1}=(A+K)^{T} P(A+K)-P+\lambda^{*} \rho I+\left(\tau_{0}-\tau_{m}+1\right) Q_{1}+\left(\tau_{M}-\tau_{0}+2\right) Q_{2}, \Theta_{2}=-Q_{1}+\delta_{0}^{2} K_{1}^{T} P K_{1}$, $\Theta_{3}=-Q_{2}+\bar{\delta}_{0}^{2} K_{1}^{T} P K_{1}$.

From (2.4), we can conclude that

$$
\begin{gathered}
{\left[f_{i}\left(e_{i}(k)\right)-l_{i} e_{i}(k)\right] \times\left[f_{i}\left(e_{i}(k)\right)-L_{i} e_{i}(k)\right] \leq 0,} \\
{\left[g_{i}\left(e_{i}\left(k-\tau_{1}(k)\right)\right)-\omega_{i} e_{i}\left(k-\tau_{1}(k)\right)\right] \times\left[g_{i}\left(e_{i}\left(k-\tau_{1}(k)\right)\right)-W_{i} e_{i}\left(k-\tau_{1}(k)\right)\right] \leq 0,} \\
{\left[g_{i}\left(e_{i}\left(k-\tau_{2}(k)\right)\right)-\omega_{i} e_{i}\left(k-\tau_{2}(k)\right)\right] \times\left[g_{i}\left(e_{i}\left(k-\tau_{2}(k)\right)\right)-W_{i} e_{i}\left(k-\tau_{2}(k)\right)\right] \leq 0 .}
\end{gathered}
$$


It can be conclude from (B.11) that there exists $\Lambda_{1}=\operatorname{diag}\left(\lambda_{11}, \lambda_{12}, \ldots, \lambda_{1 n}\right)>0$,

$$
\begin{gathered}
\sum_{i=1}^{n} \lambda_{1 i}\left[\begin{array}{c}
e_{k} \\
F\left(e_{k}\right)
\end{array}\right]^{T}\left[\begin{array}{cc}
l_{i} L_{i} \alpha_{i} \alpha_{i}^{T} & -\frac{l_{1}+L_{i}}{2} \alpha_{i} \alpha_{i}^{T} \\
-\frac{l_{1}+L_{i}}{2} \alpha_{i} \alpha_{i}^{T} & \alpha_{i} \alpha_{i}^{T}
\end{array}\right]\left[\begin{array}{c}
e_{k} \\
F\left(e_{k}\right)
\end{array}\right] \\
=\left[\begin{array}{c}
e_{k} \\
F\left(e_{k}\right)
\end{array}\right]^{T}\left[\begin{array}{cc}
\Lambda_{1} \mathcal{C}_{1} & \Lambda_{1} \mathcal{C}_{2} \\
\Lambda_{1} \mathcal{C}_{2} & \Lambda_{1}
\end{array}\right]\left[\begin{array}{c}
e_{k} \\
F\left(e_{k}\right)
\end{array}\right] \leq 0
\end{gathered}
$$

where $\alpha_{i}$ denote a column vector having " 1 " element on its $i$ th row and zeros elsewhere. In a similar way, from (B.12) and (B.13), we get

$$
\begin{gathered}
{\left[\begin{array}{c}
e_{\tau, 1} \\
G\left(e_{\tau, 1}\right)
\end{array}\right]^{T}\left[\begin{array}{cc}
\Lambda_{2} \mathscr{\ell}_{1} & \Lambda_{2} \mathscr{\ell}_{2} \\
\Lambda_{2} \mathscr{\ell}_{2} & \Lambda_{2}
\end{array}\right]\left[\begin{array}{c}
e_{\tau, 1} \\
G\left(e_{\tau, 1}\right)
\end{array}\right] \leq 0,} \\
{\left[\begin{array}{c}
e_{\tau, 2} \\
G\left(e_{\tau, 2}\right)
\end{array}\right]^{T}\left[\begin{array}{cc}
\Lambda_{3} \mathscr{\ell}_{1} & \Lambda_{3} \mathscr{\ell}_{2} \\
\Lambda_{3} \mathscr{\ell}_{2} & \Lambda_{3}
\end{array}\right]\left[\begin{array}{c}
e_{\tau, 2} \\
G\left(e_{\tau, 2}\right)
\end{array}\right] \leq 0,}
\end{gathered}
$$

where $\Lambda_{2}=\operatorname{diag}\left(\lambda_{21}, \lambda_{22}, \ldots, \lambda_{2 n}\right)>0, \Lambda_{3}=\operatorname{diag}\left(\lambda_{31}, \lambda_{32}, \ldots, \lambda_{3 n}\right)>0$. deduce

Since $\Omega_{0}<0$ implies $\Omega_{1}+\Psi^{T} P \Psi<0$, combing (B.10) and (B.14)-(B.16), we can

$$
\begin{aligned}
& \mathbb{E}\left\{\Delta V_{k}\right\} \leq \mathbb{E}\left\{\xi_{k}^{T} \Omega_{0} \xi_{k}-\left[\begin{array}{c}
e_{k} \\
F\left(e_{k}\right)
\end{array}\right]^{T}\left[\begin{array}{cc}
\Lambda_{1} \mathcal{C}_{1} & \Lambda_{1} \mathcal{C}_{2} \\
\Lambda_{1} \mathcal{C}_{2} & \Lambda_{1}
\end{array}\right]\left[\begin{array}{c}
e_{k} \\
F\left(e_{k}\right)
\end{array}\right]\right. \\
&- {\left[\begin{array}{c}
e_{\tau, 1} \\
G\left(e_{\tau, 1}\right)
\end{array}\right]^{T}\left[\begin{array}{cc}
\Lambda_{2} \mathscr{\ell}_{1} & \Lambda_{2} \mathscr{\ell}_{2} \\
\Lambda_{2} \mathscr{\ell}_{2} & \Lambda_{2}
\end{array}\right]\left[\begin{array}{c}
e_{\tau, 1} \\
G\left(e_{\tau, 1}\right)
\end{array}\right] } \\
&\left.-\left[\begin{array}{c}
e_{\tau, 2} \\
G\left(e_{\tau, 2}\right)
\end{array}\right]^{T}\left[\begin{array}{cc}
\Lambda_{3} \mathscr{\ell}_{1} & \Lambda_{3} \mathscr{\ell}_{2} \\
\Lambda_{3} \mathscr{\ell}_{2} & \Lambda_{3}
\end{array}\right]\left[\begin{array}{c}
e_{\tau, 2} \\
G\left(e_{\tau, 2}\right)
\end{array}\right]\right\} \\
& \leq \mathbb{E}\left\{\xi_{k}^{T}\left(\Omega_{1}+\Psi^{T} P \Psi\right) \xi_{k}\right\} \\
& \triangleq \mathbb{E}\left\{\xi_{k}^{T} \tilde{\Psi} \xi_{k}\right\},
\end{aligned}
$$


where

$$
\Omega_{1}=\left[\begin{array}{cccccc}
\Xi_{1} & 0 & 0 & -\Lambda_{1} \mathcal{C}_{2} & 0 & 0 \\
* & \Xi_{2} & 0 & 0 & -\Lambda_{2} \mathcal{L}_{2} & 0 \\
* & * & \Xi_{3} & 0 & 0 & -\Lambda_{3} \mathcal{L}_{2} \\
* & * & * & -\Lambda_{1} & 0 & 0 \\
* & * & * & * & -\Lambda_{2} & 0 \\
* & * & * & * & * & -\Lambda_{3}
\end{array}\right]<0 .
$$

By applying Lemma A.2 (in the Appendix A) to (3.2), we get $\widetilde{\Psi}<0$. Let $c_{0}=\lambda_{\max }(\widetilde{\Psi})$, then $c_{0}<0$, and from (B.17), we get

$$
\mathbb{E}\left\{\Delta V_{k}\right\}=\mathbb{E}\left\{\mathbb{E}\left\{V_{k+1} \mid \mathcal{\varepsilon}_{k}\right\}-V_{k}\right\} \leq c_{0} \mathbb{E}\left|e_{k}\right|^{2}
$$

From the definition of $V_{k}$, it can be easily derived that

$$
\mathbb{E} V_{k} \leq \eta_{1} \mathbb{E}\left|e_{k}\right|^{2}+\eta_{2} \sum_{i=k-\tau_{M}}^{k-1} \mathbb{E}\left|e_{i}\right|^{2}
$$

where $\eta_{1}=\lambda_{\max }(P), \eta_{2}=\left(\tau_{0}-\tau_{m}+1\right) \lambda_{\max }\left(Q_{1}\right)+\left(\tau_{M}-\tau_{0}+2\right) \lambda_{\max }\left(Q_{2}\right)$. For any scalar $\mu>1$, (B.20) together with (B.19), implies that

$$
\begin{aligned}
\mathbb{E}\left\{\mu^{k+1} V_{k+1}-\mu^{k} V_{k}\right\} & =\mu^{k+1} \mathbb{E}\left\{\Delta V_{k}\right\}+\mu^{k}(\mu-1) \mathbb{E} V_{k} \\
& \leq h_{1}(\mu) \mu^{k} \mathbb{E}\left|e_{k}\right|^{2}+h_{2}(\mu) \sum_{i=k-\tau_{M}}^{k-1} \mu^{k} \mathbb{E}\left|e_{i}\right|^{2},
\end{aligned}
$$

where $h_{1}(\mu)=\mu c_{0}+(\mu-1) \eta_{1}, h_{2}(\mu)=(\mu-1) \eta_{2}$. Furthermore, for any integer $N \geq \tau_{M}+1$, summing up both sides of (B.21) from 0 to $N-1$ with respect to $k$, we get

$$
\mathbb{E}\left\{\mu^{N} V_{N}\right\}-\mathbb{E} V_{0} \leq h_{1}(\mu) \sum_{k=0}^{N-1} \mu^{k} \mathbb{E}\left|e_{k}\right|^{2}+h_{2}(\mu) \sum_{k=0}^{N-1} \sum_{i=k-\tau_{M}}^{k-1} \mu^{k} \mathbb{E}\left|e_{i}\right|^{2}
$$


Note that for $\tau_{m} \geq 1$,

$$
\begin{aligned}
\sum_{k=0}^{N-1} \sum_{i=k-\tau_{M}}^{k-1} \mu^{k} \mathbb{E}\left|e_{i}\right|^{2} & \leq\left(\sum_{i=-\tau_{M}}^{-1} \sum_{k=0}^{i+\tau_{M}}+\sum_{i=0}^{N-1-\tau_{M}} \sum_{k=i+1}^{i+\tau_{M}}+\sum_{i=N-\tau_{M}}^{N-1} \sum_{k=i+1}^{N-1}\right) \mu^{k} \mathbb{E}\left|e_{i}\right|^{2} \\
& \leq \tau_{M} \sum_{i=-\tau_{M}}^{-1} \mu^{i+\tau_{M}} \mathbb{E}\left|e_{i}\right|^{2}+\tau_{M} \sum_{i=0}^{N-1-\tau_{M}} \mu^{i+\tau_{M}} \mathbb{E}\left|e_{i}\right|^{2}+\tau_{M} \sum_{i=N-1-\tau_{M}}^{N-1} \mu^{i+\tau_{M}} \mathbb{E}\left|e_{i}\right|^{2} \\
& \leq \tau_{M} \mu^{\tau_{M}} \max _{-\tau_{M} \leq i \leq 0} \mathbb{E}\left|e_{i}\right|^{2}+\tau_{M} \mu^{\tau_{M}} \sum_{i=0}^{N-1} \mu^{i} \mathbb{E}\left|e_{i}\right|^{2} .
\end{aligned}
$$

Then, from (B.22) and (B.23) we have

$$
\mathbb{E}\left\{\mu^{N} V_{N}\right\} \leq \mathbb{E} V_{0}+\left[h_{1}(\mu)+\tau_{M} \mu^{\tau_{M}} h_{2}(\mu)\right] \sum_{k=0}^{N-1} \mu^{k} \mathbb{E}\left|e_{k}\right|^{2}+\tau_{M} \mu^{\tau_{M}} h_{2}(\mu) \max _{-\tau_{M} \leq i \leq 0} \mathbb{E}\left|e_{i}\right|^{2}
$$

Let $\eta_{0}=\lambda_{\min }(P)$, and $\eta=\max \left\{\eta_{1}, \eta_{2}\right\}$. Obviously, we have

$$
\mathbb{E} V_{N} \geq \eta_{0} \mathbb{E}\left|e_{N}\right|^{2}
$$

It also follows easily from (B.20) that

$$
\mathbb{E} V_{0} \leq \eta \max _{-\tau_{M} \leq i \leq 0} \mathbb{E}\left|e_{i}\right|^{2}
$$

In addition, we can verify that there exists a scalar $\mu_{0}>1$ such that

$$
h_{1}\left(\mu_{0}\right)+\tau_{M} \mu_{0}^{\tau_{M}} h_{2}\left(\mu_{0}\right)=0 .
$$

Substituting (B.25)-(B.27) into (B.24), we obtain

$$
\mathbb{E}\left|e_{N}\right|^{2} \leq \frac{1}{\mu_{0}}\left(\eta+\tau_{M} \mu_{0}^{\tau_{M}} h_{2}\left(\mu_{0}\right)\right)\left(\frac{1}{\mu_{0}}\right)^{N} \max _{-\tau_{M} \leq i \leq 0} \mathbb{E}\left|e_{i}\right|^{2}
$$

Since $N$ is an any positive integer, it can be concluded from (B.28) and Definition 2.6 that the response system (2.11) can be globally exponentially synchronized with the drive model (2.10), and this completes the proof of the theorem.

\section{Acknowledgments}

The authors appreciate the editor's work and the reviewer's helpful comments and suggestions. This work was jointly supported by the National Natural Science Foundation of China under Grant no. 11072059 and the Natural Science Foundation of Jiangsu Province of China under Grant no. BK2009271. 


\section{References}

[1] L. M. Pecora and T. Carroll, "Synchronization in chaotic systems," Physical Review Letters, vol. 64, no. 8, pp. 821-824, 1990.

[2] T. L. Carroll and L. M. Pecora, "Synchronizing chaotic circuits," IEEE Transactions on Circuits and Systems, vol. 38, no. 4, pp. 453-456, 1991.

[3] J. Lü and G. Chen, "A time-varying complex dynamical network model and its controlled synchronization criteria," IEEE Transactions on Automatic Control, vol. 50, no. 6, pp. 841-846, 2005.

[4] J. Liang, Z. Wang, and X. Liu, "Exponential synchronization of stochastic delayed discrete-time complex networks," Nonlinear Dynamics, vol. 53, no. 1-2, pp. 153-165, 2008.

[5] J. Liang, Z. Wang, Y. Liu, and X. Liu, "Global synchronization control of general delayed discretetime networks with stochastic coupling and disturbances," IEEE Transactions on Systems, Man, and Cybernetics, Part B, vol. 38, no. 4, pp. 1073-1083, 2008.

[6] C. W. Wu, "Synchronization in arrays of coupled nonlinear systems with delay and nonreciprocal time-varying coupling," IEEE Transactions on Circuits and Systems II, vol. 52, no. 5, pp. 282-286, 2005.

[7] J. Zhou and T. Chen, "Synchronization in general complex delayed dynamical networks," IEEE Transactions on Circuits and Systems. I, vol. 53, no. 3, pp. 733-744, 2006.

[8] H. Gao, J. Lam, and G. Chen, "New criteria for synchronization stability of general complex dynamical networks with coupling delays," Physics Letters, Section A, vol. 360, no. 2, pp. 263-273, 2006.

[9] J. Cao, P. Li, and W. Wang, "Global synchronization in arrays of delayed neural networks with constant and delayed coupling," Physics Letters, Section A, vol. 353, no. 4, pp. 318-325, 2006.

[10] W. Lu and T. Chen, "Synchronization of coupled connected neural networks with delays," IEEE Transactions on Circuits and Systems. I, vol. 51, no. 12, pp. 2491-2503, 2004.

[11] T. Huang, C. Li, and X. Liao, "Synchronization of a class of coupled chaotic delayed systems with parameter mismatch," Chaos, vol. 17, no. 3, article 033121, p. 5, 2007.

[12] J. Cao, H. X. Li, and Daniel W. C. Ho, "Synchronization criteria of Lur'e systems with time-delay feedback control," Chaos, Solitons and Fractals, vol. 23, no. 4, pp. 1285-1298, 2005.

[13] W. Yu and J. Cao, "Synchronization control of stochastic delayed neural networks," Physica A, vol. 373, pp. 252-260, 2007.

[14] J. Cao and J. Lu, "Adaptive synchronization of neural networks with or without time-varying delay," Chaos, vol. 16, no. 1, article 013133, p. 6, 2006.

[15] Y. Tang, S. Y. S. Leung, W. K. Wong, and J. A. Fang, "Impulsive pinning synchronization of stochastic discrete-time networks," Neurocomputing, vol. 73, no. 10-12, pp. 2132-2139, 2010.

[16] T. Huang, C. Li, W. Yu, and G. Chen, "Synchronization of delayed chaotic systems with parameter mismatches by using intermittent linear state feedback," Nonlinearity, vol. 22, no. 3, pp. 569-584, 2009.

[17] Y. Sun, J. Cao, and Z. Wang, "Exponential synchronization of stochastic perturbed chaotic delayed neural networks," Neurocomputing, vol. 70, no. 13-15, pp. 2477-2485, 2007.

[18] W. Lin and Y. He, "Complete synchronization of the noise-perturbed Chua's circuits," Chaos, vol. 15, no. 2, Article ID 023705, pp. 1-9, 2005.

[19] S. Mohamad and K. Gopalsamy, "Exponential stability of continuous-time and discrete-time cellular neural networks with delays," Applied Mathematics and Computation, vol. 135, no. 1, pp. 17-38, 2003.

[20] Z. Toroczkai, "Complex networks: the challenge of interaction topology," Los Alamos Science, vol. 29, pp. 94-109, 2005.

[21] Y. Liu, Z. Wang, J. Liang, and X. Liu, "Synchronization and state estimation for discrete-time complex networks with distributed delays," IEEE Transactions on Systems, Man, and Cybernetics, Part B, vol. 38, no. 5, pp. 1314-1325, 2008

[22] Z. Wang, Y. Wang, and Y. Liu, “Global synchronization for discrete-time stochastic complex networks with randomly occurred nonlinearities and mixed time delays," IEEE Transactions on Neural Networks, vol. 21, no. 1, pp. 11-25, 2010.

[23] Z. Zhang, S. Mou, J. Lam, and H. Gao, “New passivity criteria for neural networks with time-varying delay," Neural Networks, vol. 22, no. 7, pp. 864-868, 2009.

[24] S. Mou, H. Gao, J. Lam, and W. Qiang, "A new criterion of delay-dependent asymptotic stability for Hopfield neural networks with time delay," IEEE Transactions on Neural Networks, vol. 19, no. 3, pp. 532-535, 2008.

[25] A. Ray, "Output feedback control under randomly varying distributed delays," Journal of Guidance, Control, and Dynamics, vol. 17, no. 4, pp. 701-711, 1994. 
[26] K. Hirasawa, S. Mabu, and J. Hu, "Propagation and control of stochastic signals through universal learning networks," Neural Networks, vol. 19, no. 4, pp. 487-499, 2006.

[27] Z. Wang, F. Yang, D. W. C. Ho, and X. Liu, "Robust $\mathrm{H}_{\infty}$ filtering for stochastic time-delay systems with missing measurements," IEEE Transactions on Signal Processing, vol. 54, no. 7, pp. 2579-2587, 2006.

[28] Z. Wang, D. W. C. Ho, and X. Liu, "Robust filtering under randomly varying sensor delay with variance constraints," IEEE Transactions on Circuits and Systems II, vol. 51, no. 6, pp. 320-326, 2004.

[29] D. Yue, Y. Zhang, E. Tian, and C. Peng, "Delay-distribution-dependent exponential stability criteria for discrete-time recurrent neural networks with stochastic delay," IEEE Transactions on Neural Networks, vol. 19, no. 7, pp. 1299-1306, 2008.

[30] Y. Zhang, D. Yue, and E. Tian, "Robust delay-distribution-dependent exponential stability criteria of discrete-time stochastic neural networks with time-varying delays," Neurocomputing, vol. 72, pp. 1265-1273, 2009.

[31] Y. Zhao, H. Gao, J. Lam, and KE. Chen, "Stability analysis of discrete-time recurrent neural networks with stochastic delay," IEEE Transactions on Neural Networks, vol. 20, no. 8, pp. 1330-1339, 2009.

[32] Y. Liu, Z. Wang, and X. Liu, "Global exponential stability of generalized recurrent neural networks with discrete and distributed delays," Neural Networks, vol. 19, no. 5, pp. 667-675, 2006.

[33] E. N. Sanchez and J. P. Perez, "Input-to-state stability (ISS) analysis for dynamic neural networks," IEEE Transactions on Circuits and Systems. I, vol. 46, no. 11, pp. 1395-1398, 1999.

[34] S. Boyd, L. El Ghaoui, E. Feron, and V. Balakrishnan, Linear Matrix Inequalities in System and Control Theory, vol. 15 of SIAM Studies in Applied Mathematics, SIAM, Philadelphia, Pa, USA, 1994. 


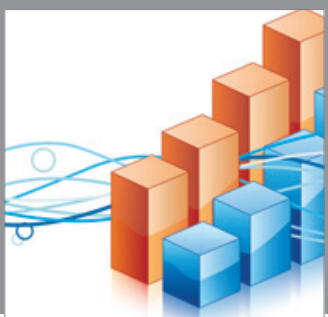

Advances in

Operations Research

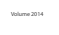

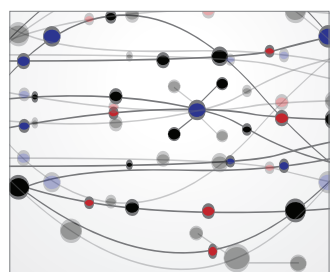

\section{The Scientific} World Journal
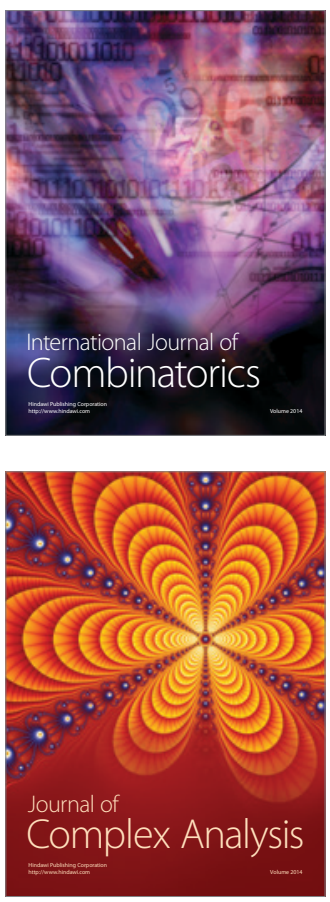

International Journal of

Mathematics and

Mathematical

Sciences
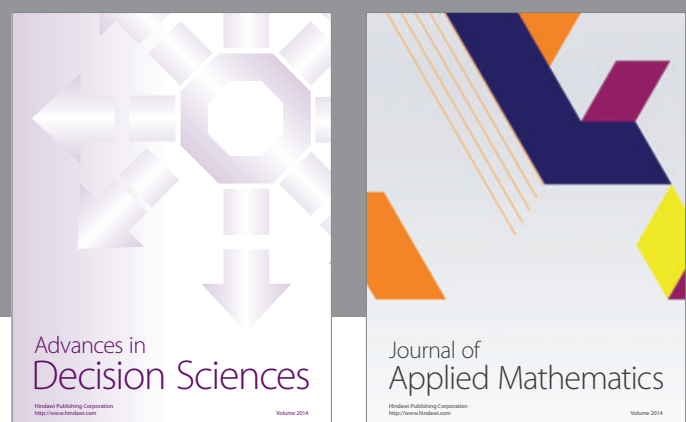

Journal of

Applied Mathematics
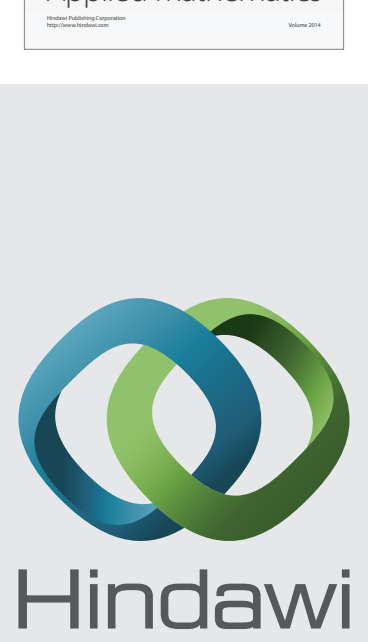

Submit your manuscripts at http://www.hindawi.com
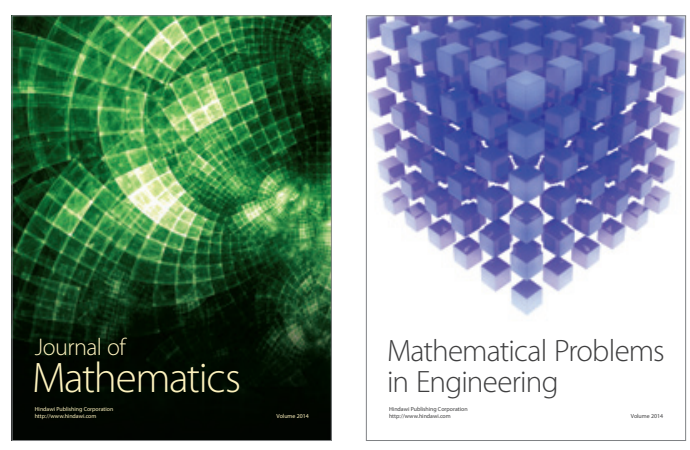

Mathematical Problems in Engineering
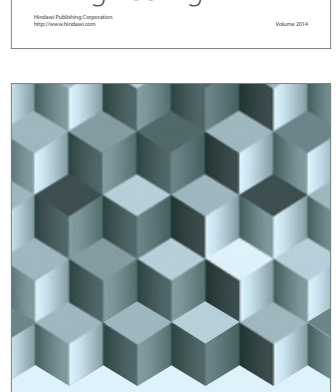

Journal of

Function Spaces
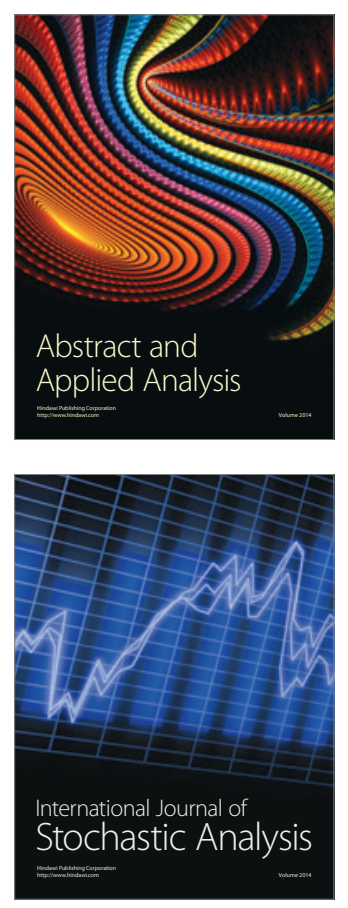

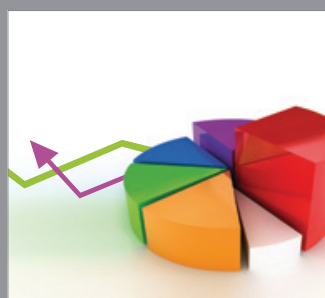

ournal of

Probability and Statistics

Promensencen
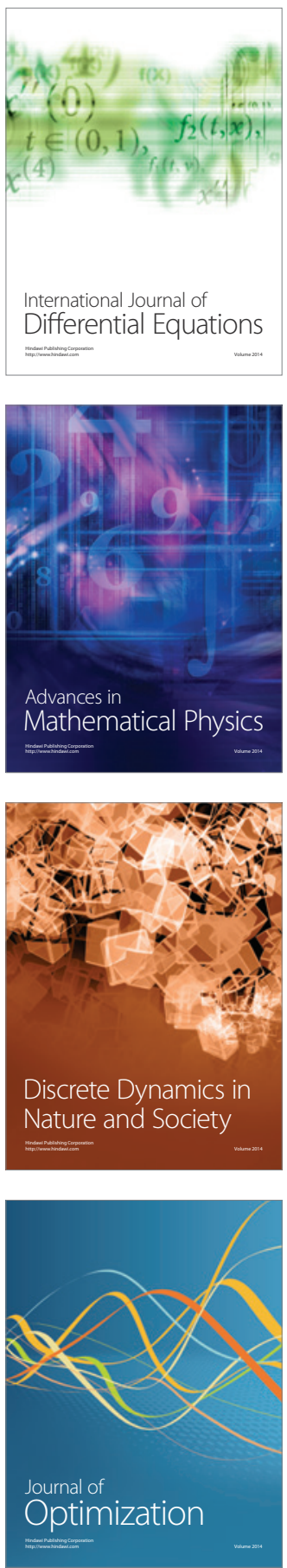